The Astrophysical Journal, 682:586-592, 2008 July 20

(C) 2008. The American Astronomical Society. All rights reserved. Printed in U.S.A.

\title{
MOST SPACE-BASED PHOTOMETRY OF THE TRANSITING EXOPLANET SYSTEM HD 209458: TRANSIT TIMING TO SEARCH FOR ADDITIONAL PLANETS
}

\author{
ELIZA Miller-RicCI \\ Harvard-Smithsonian Center for Astrophysics, 60 Garden Street, Cambridge, MA 02138; emillerricci@cfa.harvard.edu \\ JASON F. Rowe \\ University of British Columbia, 6224 Agricultural Road, Vancouver, BC V6T 1Z1, Canada \\ Dimitar Sasselov \\ Harvard-Smithsonian Center for Astrophysics, 60 Garden Street, Cambridge, MA 02138 \\ JAYMie M. MatThews \\ University of British Columbia, 6224 Agricultural Road, Vancouver, BC V6T 1Z1, Canada \\ DAVID B. GuENTHER \\ Department of Astronomy and Physics, St. Mary's University, Halifax, NS B3H 3C3, Canada \\ RAINER KUSCHNIG \\ Department of Physics and Astronomy, University of British Columbia, 6224 Agricultural Road, Vancouver, BC V6T 1Z1, Canada \\ Anthony F. J Moffat \\ Département de Physique, Université de Montréal, C.P. 6128, Succ. Centre-Ville, Montréal, QC H3C 3J7, Canada \\ SLAVEK M. RucinsKI \\ David Dunlap Observatory, University of Toronto, P.O. Box 360, Richmond Hill, ON L4C 4Y6, Canada \\ Gordon A. H WalKer \\ Department of Physics and Astronomy, University of British Columbia, \\ 6224 Agricultural Road, Vancouver, BC V6T 1Z1, Canada \\ AND \\ WERNER W. WeISS \\ Institut für Astronomie, Universität Wien, Türkenschanzstrasse 17, A-1180 Wien, Austria \\ Received 2006 October 17; accepted 2008 January 30
}

\begin{abstract}
We report on the measurement of transit times for the HD 209458 planetary system from photometry obtained with the MOST (Microvariability and Oscillations of Stars) space telescope. Deviations from a constant orbital period can indicate the presence of additional planets in the system that are yet undetected, potentially with masses approaching an Earth mass. The MOST data sets of HD 209458 from 2004 and 2005 represent unprecedented time coverage with nearly continuous observations spanning 14 and 43 days and monitoring three transits and 12 consecutive transits, respectively. The transit times that we obtain show no variations on three scales: (1) no long-term change in $P$ since before 2004 at $25 \mathrm{~ms}$ level, (2) no trend in transit timings during the 2005 run, and (3) no individual transit timing deviations above $80 \mathrm{~s}$ level. Together with previously published transit times from Agol \& Steffen, this allows us to place limits on the presence of additional close-in planets in the system, in some cases down to below an Earth mass. This result, along with previous radial velocity work, now eliminates the possibility that a perturbing planet could be responsible for the additional heat source needed to explain HD 209458b's anomalous low density.
\end{abstract}

Subject headings: methods: data analysis — planetary systems — stars: individual (HD 209458)

Online material: color figures

\section{INTRODUCTION}

The search for extrasolar planets has been characterized recently by a move toward the discovery of lower mass planets compared to the gas giant planets of our solar system. While the techniques of radial velocities, transits, and microlensing have already proved to be successful in detecting extrasolar planets, the novel idea of using transit timing variations (TTVs) to detect additional low-mass companions in known transiting planetary systems (Agol et al. 2005; Holman \& Murray 2005) is a recent and exciting addition to the repertoire of planet detection methods, which has not yet been widely applied. The premise of the
TTV method is that a planet known to transit its host star will do so at a near-constant period unless there is an additional planet in the system to perturb its orbit. In the case of a second planet in the system, the observer will then view transits that deviate from a constant period on a level of seconds to minutes, depending on the mass and orbital parameters of the perturbing planet. An analysis of transit timings in the TrES-1 system was carried out by Steffen \& Agol (2005), placing useful limits on the presence of additional planets around that star. They determine that a $(2: 1$ resonant) companion planet in that system would generally need to have a mass comparable to or less than an Earth mass. A subsequent analysis performed on HST observations of HD 209458 
by Agol \& Steffen (2007) places similar limits on the presence of companion planets in that system. The addition of the MOST ${ }^{1}$ data now allows for more stringent limits to be placed on such planets.

HD 209458 was the first star found to have a transiting planet (Charbonneau et al. 2000; Henry et al. 2000) and remains the second brightest star known to have a transiting planetary companion, making it suitable for a large number of follow-up observations. As more transiting planets are discovered, it is becoming clear that HD 209458b has an anomalously large radius for its mass when compared with theoretical predictions (Laughlin et al. 2005c). Speculation as to the mechanism that is keeping the planet "too large" has led to several theories including (1) additional sources of internal heating such as strong winds (Guillot \& Showman 2002), (2) obliquity tides due to a disalignment of the planetary spin axis and the orbit normal (Winn \& Holman 2005), and (3) the presence of additional low-mass planets in the system inducing eccentricity in the orbit of HD 209458b and causing ongoing tidal heating (Bodenheimer et al. 2001, 2003). The first of these theories seems less likely, seeing as it would apply to the radii of all of the transiting planets. The latter two have remained viable theories in explaining the anomalous radius of HD 209458b.

The TTV analysis that follows serves to shed some light on the presence of additional planets in the system and, together with previous observations, now effectively rules out the third option listed above. The Microvariability and Oscillations of Stars (MOST) satellite (Walker et al. 2003; Matthews et al. 2004) observed HD 209458 in 2004 and 2005 as it passed through the satellite's continuous viewing zone (CVZ). A combined total of 15 complete transits were observed, with 12 of these being consecutive transits in 2005. The combination of the timings for these transits along with previously published transit times by Agol \& Steffen (2007) allows us to place strong limits on the presence of additional planets in the system, which would be undetectable by other currently available techniques.

\section{MOST PHOTOMETRY}

Photometry of HD 209458 was obtained with MOST during 13.5 days in 2004 and 42.9 days in 2005. MOST houses a $15 \mathrm{~cm}$ optical telescope feeding a CCD photometer through a single custom broadband optical filter. From its $820 \mathrm{~km}$ Sun-synchronous polar orbit it can monitor stars passing through its CVZ for up to 2 months without interruption. MOST can collect photometry in several operating modes, including "direct imaging," in which a target star is centered in a subraster on the MOST Science CCD for a combination of aperture and PSF (point-spread function) photometry.

HD 209458 was observed in direct imaging mode, with exposures of $1.5 \mathrm{~s}$ (as outlined in Rowe et al. 2006). The point-topoint precision for these observations depends on the level of stray light scattered into the instrument and can be as low as $3 \mathrm{mmag}$ at times of very little stray light and as high as $20 \mathrm{mmag}$ during instances of high stray light. At low levels of stray light this corresponds to a photometric error that is essentially equal to the Poisson noise limit, whereas during times of increased scattered light this can degrade to up to 1.5 times the Poisson limit (see Fig. 2 in Rowe et al. 2007). For the 2004 observations the sampling rate was varied to obtain a higher rate during phases of the known planet's secondary eclipse, resulting in an average sampling of five individual exposures per minute. For the 2005 pho-

${ }^{1}$ Based on data from the MOST satellite, a Canadian Space Agency mission, jointly operated by Dynacon, Inc., the University of Toronto Institute for Aerospace Studies, and the University of British Columbia, with the assistance of the University of Vienna.
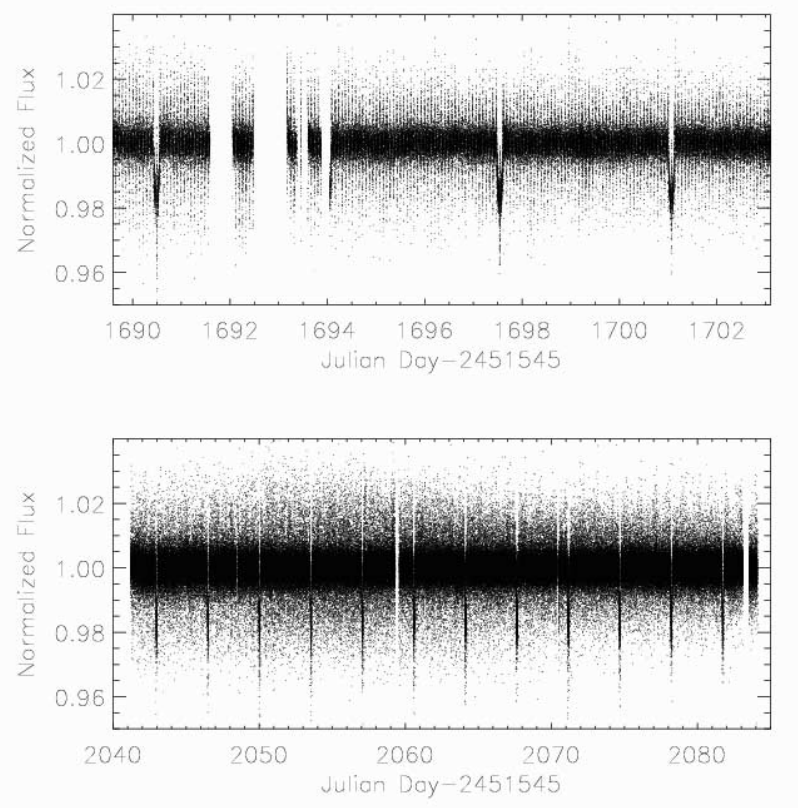

FIG. 1.-Unbinned MOST HD 209458 photometry from 2004 (top) and 2005 (bottom). Note the difference in time axis scales between the two panels. Incidences of very high stray light and passages through the SAA have been removed. Gaps in the light curve during 2004 are due to onboard crashes during new software testing, while the two shorter gaps in 2005 are caused by data removed due to particularly high cosmic-ray fluxes.

tometry, the sampling rate was 6 times per minute, a limit set by the data downlink capacity of the MOST satellite in the direct imaging mode.

The reduction procedures for the 2004 photometry are described by Rowe et al. (2006) and include corrections for stray light due to earthshine modulated with the satellite orbit. In the 2005 data there was electronic "crosstalk" between the timing of the Science CCD and the Attitude Control System (ACS) CCD onboard electronics, with a timescale of about 3 days. This manifested itself as a subtle band of noise moving across the CCD subraster in about half a day. Such crosstalk added a non-Poisson component to the photometric noise and, uncorrected, could induce deviations of about $1 \mathrm{mmag}$ on timescales of up to a day. By comparing the standard deviation of the background sky counts in the subraster against the square root of the mean sky count we can trace the occurrence of crosstalk. Corrections are then applied to the sky counts and the instrumental magnitudes of HD 209458. Other than this, the 2004 and 2005 data sets have been uniformly reduced.

For this analysis we have removed from the data set incidences of very high levels of stray light (earthshine) modulated at the satellite's orbital period, as well as times of passage through the South Atlantic Anomaly (SAA) when the cosmic-ray flux is high, and other instances of severe cosmic-ray hits. This reduces the total number of observations by about $12 \%$ but does not seriously reduce the coverage of the observations over the HD $209458 \mathrm{~b}$ orbital period. The reduced 2004 and 2005 light curves are shown in Figure 1.

The pronounced gaps seen in Figure 1 in the 2004 data are due to onboard crashes caused by the testing of new software during those trial observations. The two shorter noticeable gaps in the 2005 light curve are due to especially severe SAA passages, which were removed from the data. There is some evolution of the level of scattered light during the 2005 data, dropping slightly toward the end of the run, consistent with seasonal evolution over 
TABLE 1

Orbital and Physical Parameters FOR HD 209458

\begin{tabular}{|c|c|}
\hline Parameter & Value \\
\hline$M *\left(M_{\odot}\right) \ldots \ldots \ldots \ldots \ldots$ & $1.101 \pm 0.064^{\mathrm{a}}$ \\
\hline$R_{*}\left(R_{\odot}\right) \ldots \ldots \ldots \ldots \ldots \ldots$ & $1.118 \pm 0.03$ \\
\hline$R_{\mathrm{pl}}\left(R_{\mathrm{Jup}}\right) \ldots \ldots \ldots \ldots \ldots$ & $1.339 \pm 0.04$ \\
\hline 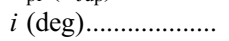 & $86.929 \pm 0.01^{\mathrm{a}}$ \\
\hline 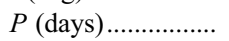 & $3.52474832 \pm 0.00000029$ \\
\hline 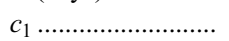 & 0.410769 \\
\hline$c_{2} \ldots \ldots \ldots \ldots \ldots \ldots \ldots \ldots \ldots$ & -0.108909 \\
\hline$c_{3} \ldots \ldots \ldots \ldots \ldots \ldots$ & 0.904020 \\
\hline$c_{4} \ldots \ldots \ldots \ldots \ldots \ldots \ldots \ldots$ & -0.437364 \\
\hline
\end{tabular}

NoтE.-The nonlinear limb-darkening coefficients calculated for the MOST bandpass are given by $c_{1}-c_{4}$.

${ }^{\mathrm{a}}$ From Knutson et al. (2007).

6 weeks that has been observed for other targets. There is also an unfortunate coincidence for MOST observations of HD 209458, in that the giant planet orbits at a period that is almost exactly 50 times the 101.413 minute orbital period of MOST. Hence, when we remove portions of the light curve with high stray light, these consistently lie at the same phases in the light curve phased to the planet transit period. We are alert to the possible effects on the transit timing analysis and have found that our results are not very sensitive to this effect (see below).

\section{TRANSIT TIMES FOR HD 209458B}

In computing our transit times we compare the MOST data against a model transit curve, constructed using the formalism set forth in Mandel \& Agol (2002) for a source with nonlinear limb darkening. To determine the orbital parameters for the model we start with those laid out in Knutson et al. (2007), which in turn were determined by a multiparameter fit to data from the STIS spectrometer aboard the Hubble Space Telescope (HST). Noting that the MOST transit light curve appears to be slightly deeper than this model would predict, we then fit for the stellar and planetary radii by minimizing the $\chi^{2}$ statistic for both variables on the composite phased and binned MOST light curve. Our values for the two radii differ somewhat from the HST light curve but lie within the $1 \sigma$ error bars determined for that data set, with the MOST data implying a planetary radius of $1.339 R_{\text {Jup }}$ and a stellar radius of $1.118 R_{\odot}$ (see discussion in Rowe et al. 2007). Nonlinear limb-darkening parameters for the MOST bandpass are derived from synthetic spectra calculated by R. Kurucz. ${ }^{2}$ This is accomplished by summing the spectra across the disk of the star then multiplying by the throughput of the MOST optics and detector. The resultant transit model has system parameters that are given in Table 1, and this model is overlaid on the phased and binned data in Figure 2. We note that some correlation in the residuals from this model (red noise) remains. This is most likely due to the stray light experienced by MOST, as it repeats at approximately the orbital period of the satellite, despite reduction procedures to correct for this effect. We pay particularly close attention to the modulation of stray light to determine its effects on the transit timing measurements as described below.

To determine times for each of the individual transits, the model light curve is computed at impact parameters corresponding with each of the MOST data points. We then find the centerof-transit time for each transit at which the $\chi^{2}$ value for the fit to the data is minimized. The resultant times are presented in

\footnotetext{
${ }^{2}$ See http://kurucz.harvard.edu/stars/hd209458.
}

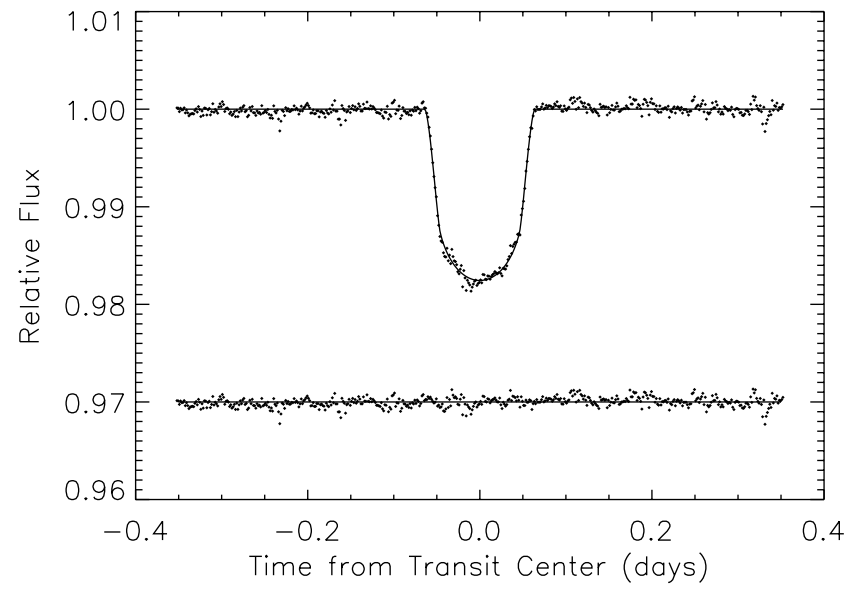

FIg. 2.-Combined 2004 and 2005 data overlaid with the transit model (above) and residuals from this model (below). The data have been phased at the orbital period of the planet and binned at a 2 minute interval.

Table 2 in HJD, calculated using the IDL function helio_jd (Landsman 1993). We measured transit times for each of the three complete transits in the 2004 data and the 12 transits in 2005 , then calculated the timing difference from the expected times of transit, as shown in Figures 3 and 4. The expected times of transit are calculated by adding the correct number of orbital periods of HD 209458 b to the 2003 ephemeris from Knutson et al. (2007). We note that our data agree with the orbital period and ephemeris of this previous work, since our data points in Figures 3 and 4 lie almost equally above and below the line representing the expected times $(O-C=0)$. As a reference, in Figure 3 we also show the times for the four transits observed in 2003 with STIS as reported by Agol \& Steffen (2007), who used a similar method to determine transit times.

Our error bars are calculated using a bootstrapping Monte Carlo simulation similar to the one described in Agol \& Steffen (2007). For each transit we shift the residuals from the bestfit transit model (and their associated errors) by a random number of points. We then add the new residuals back onto the transit model and recalculate the center-of-transit time using the same procedure described above, thus maintaining the

TABLE 2

Best-Fit Transit Times

\begin{tabular}{|c|c|c|c|}
\hline Transit No. & $T_{C}(\mathrm{HJD})$ & $\sigma(\mathrm{HJD})$ & Reduced $\chi^{2}$ \\
\hline 1 & $2,453,235.49852$ & \pm 0.00068 & 0.83 \\
\hline 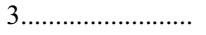 & $2,453,242.54809$ & \pm 0.00049 & 0.74 \\
\hline 4.................... & $2,453,246.07367$ & \pm 0.00061 & 0.84 \\
\hline $101 \ldots \ldots \ldots \ldots$ & $2,453,587.97480$ & \pm 0.00074 & 0.91 \\
\hline $102 \ldots \ldots \ldots \ldots \ldots$ & $2,453,591.49958$ & \pm 0.00051 & 0.82 \\
\hline $103 \ldots \ldots \ldots \ldots$ & $2,453,595.02365$ & \pm 0.00060 & 0.88 \\
\hline $104 \ldots \ldots$ & $2,453,598.54930$ & \pm 0.00053 & 0.79 \\
\hline $105 \ldots \ldots \ldots \ldots \ldots \ldots$ & $2,453,602.07253$ & \pm 0.00062 & 0.89 \\
\hline $106 \ldots \ldots \ldots \ldots \ldots \ldots$ & $2,453,605.59805$ & \pm 0.00055 & 0.81 \\
\hline 107...................... & $2,453,609.12212$ & \pm 0.00056 & 0.91 \\
\hline $108 \ldots \ldots \ldots \ldots \ldots \ldots$ & $2,453,612.64690$ & \pm 0.00056 & 0.75 \\
\hline $109 \ldots \ldots \ldots \ldots \ldots$ & $2,453,616.17165$ & \pm 0.00062 & 0.82 \\
\hline $110 \ldots$ & $2,453,619.69667$ & \pm 0.00065 & 0.79 \\
\hline $111 \ldots \ldots \ldots \ldots \ldots \ldots$ & $2,453,623.22186$ & \pm 0.00075 & 0.91 \\
\hline 112 & $2,453,626.74617$ & \pm 0.00079 & 1.02 \\
\hline
\end{tabular}

NoтE.-Transit times for three transits in 2004 and 12 transits in 2005. Transit 2 was not fully observed due to an onboard software crash, and it has been omitted, since an accurate transit time cannot be obtained. 


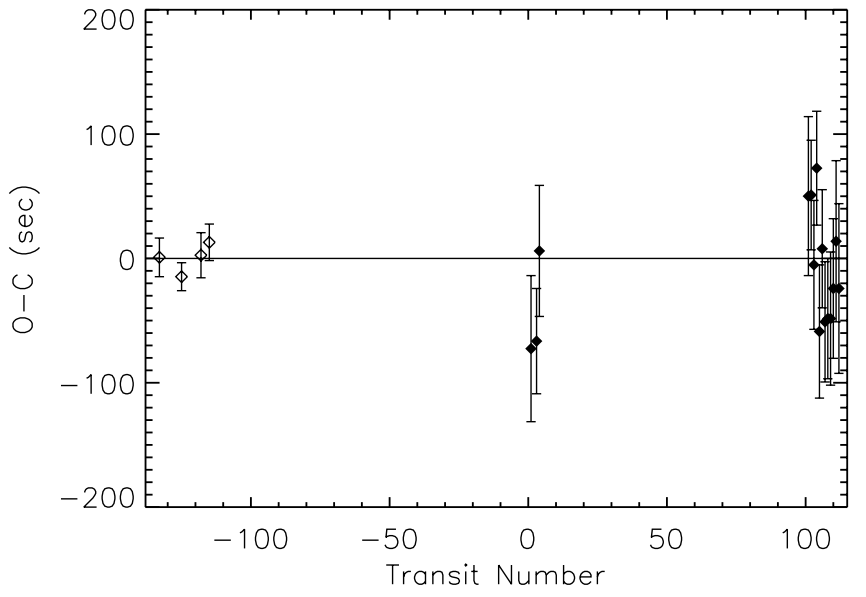

FIG. 3.-Deviation from predicted time of transit vs. transit number for the 2004 and 2005 MOST data ( filled symbols) and for the 2003 HST data as reported by Agol \& Steffen (2007; open symbols). The expected time of transit is based on the ephemeris of Knutson et al. (2007).

original point-to-point correlations. The goal realized by this error analysis is to understand the effects of correlated noise in the MOST light curve due to the repeating pattern of stray light. The resulting error bars in Figures 3 and 4 are somewhat larger than those obtained from a simple $\chi^{2}$ analysis (generally by less than a factor of 2 , but occasionally by a factor of several). In addition, we have performed tests of our sensitivity to the modulation of stray light during the MOST satellite orbit by applying more stringent removal of times of higher stray light. This does not have a significant effect on the transit times that we determine and only unnecessarily reduces the overall duty cycle of the observations. It must also be noted that, at the precision and time coverage of the MOST data, intrinsic low-amplitude variations in the star HD 209458 a may be present. This may represent a fundamental limit in the systematic errors of the timing data for this system.

Long-term and periodic trends in the data not associated with the HD 209458b orbit could be a serious problem in limiting the accuracy of our transit times, so we have examined this issue closely. This is especially true if a deviation due to instrumental or satellite orbital effects was to occur during transit ingress or egress. As an example, consider a transit light curve in which ingress has not been correctly normalized so all of the points are reported at a flux level that is slightly too low. In this case, our analysis method would report this transit as occurring earlier than it actually did. We have carefully identified and corrected for possible long-term and periodic artifacts in the data to produce a properly normalized light curve, as described in Rowe et al. (2006). These include (1) dips and rises in the light curve stemming from electronic crosstalk as described in $\S 2$; (2) cycle-perday modulation of stray earthshine due to the Sun-synchronous orbit of MOST; and (3) filtering of longer term trends in the data that might be instrumental, stellar, or a combination of both.

Another consideration in determining transit times is the importance of having a light curve that is well sampled during ingress and egress, as this is where the timing signal is most sensitive. In the case of a partial transit that is missing either ingress or egress, the value adopted for orbital inclination (which determines the transit duration) in the model light curve must be finely tuned to the true value. Otherwise, the risk is that partially sampled transits could have large systematic errors in their measured timings. For the transit times reported from the MOST data only complete transits were used. In particular, the second transit from

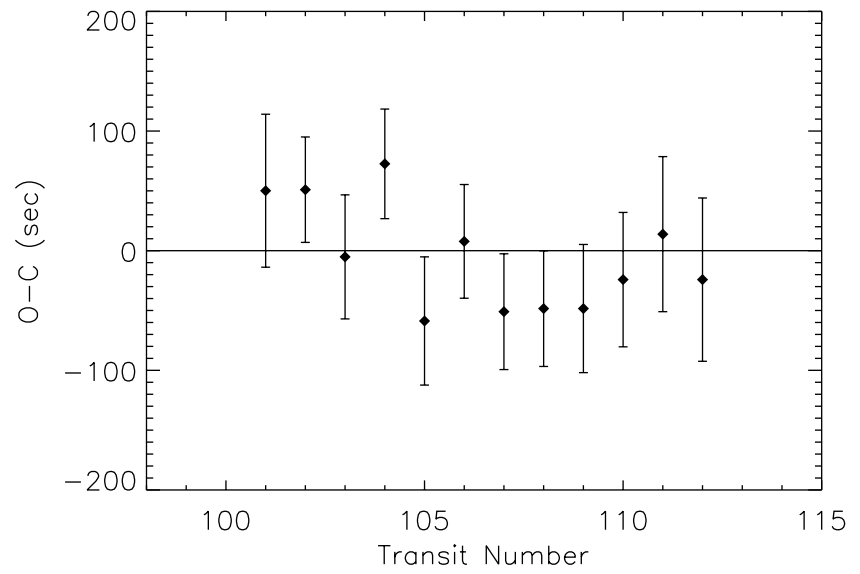

FIG. 4.-Deviation from predicted time of transit vs. transit number for the 12 transits in the 2005 data only. The short-term scatter in the transit times here is always less than $100 \mathrm{~s}$ and is less than $30 \mathrm{~s}$ for the last seven transits, where scattered light entering the instrument is at a minimum.

the 2004 observations is not used in this analysis, since data are only available for half of it. Note that the effect of removing short intervals of very high stray light (which often affects ingress more than egress or vice versa) is negligible, since only a small number of data points are actually removed from each transit.

\section{LIMITS ON OTHER CLOSE-IN PLANETS IN THE HD 209458 SYSTEM}

The two seasons of transit timing data from MOST allow us to look for variations due to several effects: (1) orbital decay of planet HD 209458b (Sasselov 2003), (2) precession of its orbit (Miralda-Escudé 2002; Laughlin et al. 2005a; Heyl \& Gladman 2007), (3) moons (Sartoretti \& Schneider 1999; Brown et al. 2001), and (4) orbit perturbations caused by additional planets in the system (Agol et al. 2005; Holman \& Murray 2005; Steffen 2006). The first two effects would cause long-term variations in the observed time between successive transits. To check for these types of long-term drifts, our best-fit times for the 15 observed transits from MOST can be combined with the 13 transit times reported by Agol \& Steffen (2007) to determine a revised orbital period for HD 209458b. Fitting an orbital period to these 28 transit times spanning from 2000 April to 2005 August yields a period of $3.52474832 \pm 0.00000029$ days. This is in agreement with the 2003 orbital period reported by Knutson et al. (2007) to within $25 \mathrm{~ms}$ (within their $1 \sigma$ error bars of $33 \mathrm{~ms}$ ). The expected drift in the orbital period of HD 209458b due to the first two effects in the list above over the $2 \mathrm{yr}$ baseline obtained when comparing our orbital period to the 2003 period of Knutson et al. (2007) remains smaller than the current sensitivity.

The latter two effects on the list presented above will cause short-term timing variations. In terms of effect 4 , we set our approach to analyzing HD 209458 as follows. Current Doppler radial velocity (RV) data already exclude planets with $M>3 \times$ $10^{-4} M_{\odot}\left(100 M_{\oplus}\right)$ and $P<100$ days (Laughlin et al. $2005 \mathrm{~b}$ ), and at such long-period orbits RVs are more sensitive than TTVs. This interplay between RV and TTV limits was pointed out by Agol et al. (2005, their Fig 7) and is likely to be common to other bright transiting systems with good RV data (e.g., HD 189733, TrES-1, HAT-P-1). Therefore, the set of MOST transit times is particularly fit to place limits on small close-in perturbing planets, with $P<15$ days (or about one third of the length of the observing run). Despite its limitations, this is of great interest for the HD 209458 system, because such small close-in planets could still provide enough forcing to the eccentricity of HD 


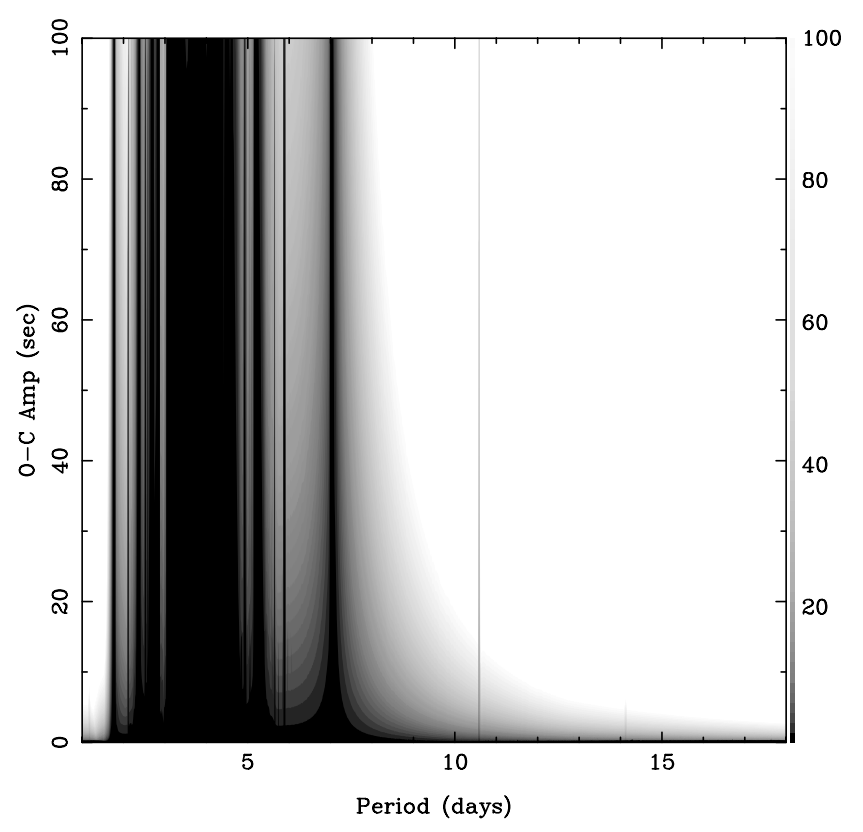

FIG. 5.- $N$-body results for maximum transit timing deviation vs. orbital period of the perturbing planet. The color scale as defined on the right side of the plot indicates the mass of the perturbing planet (in $M_{\oplus}$ ). The 15 MOST transits show no timing deviations above the level of $100 \mathrm{~s}$. The last seven transits observed are consistent with a constant orbital period at the level of $30 \mathrm{~s}$. [See the electronic edition of the Journal for a color version of this figure.]

$209458 \mathrm{~b}$ for tidal heating to occur. The same is true for systems like HAT-P-1b.

Our results show no evidence for short-term timing variations. The short-term scatter in the data is always below $100 \mathrm{~s}$ and is below $30 \mathrm{~s}$ for the last seven transits in 2005. This imposes strong constraints on any additional planets - in both mass and orbits. To determine what types of planets are ruled out by the MOST data we solved the classical $N$-body problem,

$$
\frac{d^{2} x_{i}}{d t^{2}}=-\sum_{j=1 ; j \neq i} N \frac{G m_{j}\left(x_{i}-x_{j}\right)}{\left|x_{i}-x_{j}\right|^{3}}
$$

where for three bodies, $x$ describes the initial positions of the particles. For the HD 209458 system, we assumed a stellar mass of $1.101 M_{\odot}$ and planetary mass of $0.69 M_{\text {Jup }}$ in a circular orbit with a period of 3.52474832 days. A third body was inserted with an initially circular orbit, with periods ranging from 1 to 18 days in increments of 0.01 days and masses from 1 to $100 M_{\oplus}$ in $1 M_{\oplus}$ increments, and on coplanar orbits to HD 209458b. The solution was advanced at $1.0 \mathrm{~s}$ intervals for 1000 orbits of HD $209458 \mathrm{~b}(\sim 3 \times$ $10^{8} \mathrm{~s}$ ) using the LSODA routine from ODEPACK (Radhakrishnan \& Hindmarsh 1993). In the case where the two planets in the simulation passed within $1 R_{\mathrm{Jup}}$ of each other, we determined that a collision had taken place, and the $N$-body code was terminated. These orbits were deemed unstable over the long term. Resultant $O-C$ values from the simulations were calculated by a linear interpolation to estimate the integration time when HD 209458b returns to the midpoint of crossing the disk of the star. Also, in certain resonant orbits where we found that our data could limit the masses of perturbing planets to less than $1 M_{\oplus}$, we ran a small number of additional $N$-body simulations down to $0.1 M_{\oplus}$ at 0.1 $M_{\oplus}$ intervals to determine what ranges of sub-Earth mass planets were ruled out.

To determine the expected magnitude of the TTVs, we compute a Fourier transform of the $O-C$ series for each value of

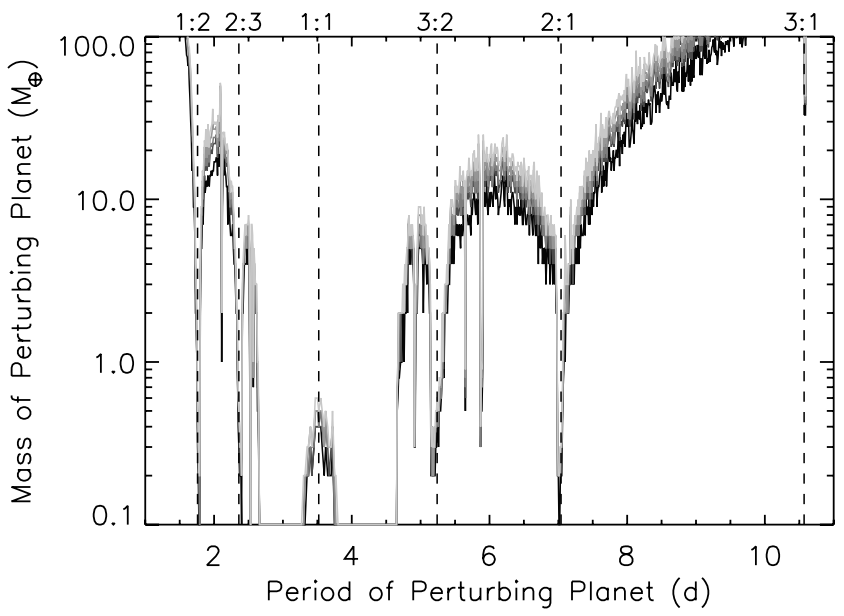

FIG. 6.-Maximum mass allowed for a perturbing planet in the HD 209458 system, which still remains consistent with the MOST and HST transit times (from this work and Agol \& Steffen 2007, respectively). The curves correspond to the masses of additional planets that are ruled out at the $60 \%$ (black), $90 \%$ (darkest gray), 95\% (dark gray), 99\% (medium gray), and 99.9\% (light gray) confidence levels, based on the $1 \sigma$ error bars on the transit timing measurements. Planets occupying the region of parameter space above the curves are ruled out by the available transit timing data. [See the electronic edition of the Journal for a color version of this figure.]

period and mass of the third body and extract the largest amplitude. This is shown in Figure 5, where we plot the TTV amplitude versus orbital period resulting from the $N$-body simulations. In addition, we compare the $N$-body results against all of the available transit timing data to determine the maximum mass that an additional planet in the HD 209458 system could possess while still remaining consistent with the data. This allows for robust mass limits to be placed on perturbing bodies in the HD 209458 system. We fit the combination of the 15 MOST transit times and the 13 $H S T$ transit times (Agol \& Steffen 2007) to each of the $O-C$ series generated by the $N$-body code. This allows for a more complete coverage of the libration period of any hypothetical two-planet system, than fitting the MOST data alone. We leave a timing offset and slope as free parameters. From this process, we determine the maximum mass of perturbing planet that remains consistent with the $1 \sigma$ error bars on the transit times as a function of the orbital period of the perturbing body, which we show in Figure 6.

From Figure 6, we place the following constraints on the presence of additional planets in the HD 209458 system, at the $99 \%$ $(3 \sigma)$ confidence limit. First, we explore possible planets in inner orbits. Placed between HD 209458b (at 0.045 AU) and the star, such planets are constrained to a very narrow range of orbits. Nonzero eccentricity is a given but is also limited for stability reasons. These orbits may be more likely due to resonant trapping during migration (Zhou et al. 2005), most likely at $1: 2$ with $P_{\text {orb }} \approx 1.76$ days. The transit timing data limit the mass of such planets to sub-Earth masses, $M<0.9 \times 10^{-6} M_{\odot}$ (about $0.3 M_{\oplus}$ ). If between the resonances, a planet could be as massive as $20 M_{\oplus}$ $\left(6 \times 10^{-5} M_{\odot}\right)$ and escape detection in the transit timing data.

Previously undetected planets in outer orbits have a larger range of possible orbits and relaxed stability requirements on their eccentricity, compared to the inner ones discussed above. Nice illustrations can be found in the detailed analysis of TrES-1 by Steffen $\&$ Agol (2005) and in Figure 5 by Agol et al. (2005). The MOST and HST transit data exclude sub-Earth mass planets down to $0.3 M_{\oplus}$ in the outer $3: 2$ and $2: 1$ mean-motion resonances, and in the $3: 1$ resonance with $M>8 \times 10^{-5} M_{\odot}\left(40 M_{\oplus}\right)$. Outside of resonances, the MOST data limit any planets with $P<7$ days to $M<17 M_{\oplus}$ and with 7 days $<P<10$ days to $M<100 M_{\oplus}$. 
The exact limits depend on the period of the perturbing planet according to Figure 6 . For orbits with periods longer than 10 days the Doppler RV limits are better.

A range of intermediate orbits surrounding the $1: 1$ resonance with the transiting planet HD 209458b are unstable for the lowest mass perturbers, resulting in ejections or collisions. Between 2.65 and 4.65 day periods we can rule out sub-Earth mass planets due to a combination of both TTV constraints and stability requirements.

In addition to the $N$-body simulations described above, we performed a limited number of additional calculations for perturbing planets in either mutually inclined orbits relative to the transiting planet, or in initially eccentric orbits. For the case of a perturbing planet on an initially circular orbit, we find that only small eccentricities are attained (generally less than 0.1 ). However, if even a small initial eccentricity or mutual inclination between the planets is added, the resulting orbit of the perturbing planet can attain a significantly higher mean eccentricity over the course of 1000 orbits. The TTVs from these configurations therefore tend to be larger than those from the case presented above, of a perturbing planet on a circular orbit, coplanar with HD 209458b. In addition, there is a smaller range of stable orbits in these cases. There are some limited cases, however, where an eccentric companion can cause slightly larger TTVs than a planet on a circular orbit. As another consideration, perturbing planets starting from different mean longitudes can result in TTVs that vary somewhat in amplitude, where we always begin our $N$-body simulations with the second planet in an orbit $90^{\circ}$ out of phase with HD 209458b. In the absence of these exceptions, the limits that we have placed on additional planets in the HD 209458 system are generally robust limits across the entire range of eccentricity parameter space, due to the fact that additional planets residing in eccentric or inclined orbits tend to have even larger observable effects on the transit times of HD 209458b.

\section{SUMMARY AND CONCLUSIONS}

The addition of the $15 M O S T$ transit times to the 13 previously available $H S T$ transit times from Agol \& Steffen (2007) has allowed us to place the tightest available limits on the presence of additional planets in the HD 209458 system with orbital periods under 11 days. We rule out sub-Earth mass planets in the inner $1: 2$ and $2: 3$ resonances, as well as in the outer $2: 1$ and $3: 2$ resonances. Super-Earths are excluded in a range of intermediate orbits as shown in Figure 6.

The MOST transit timing data are unique at this time in that they have 12 consecutive transits of uniform quality and consistent photometric precision, which is very difficult to achieve from the ground for such long time spans. For HD 209458 these types of observations have only been possible from space and they have not been practical with the Hubble Space Telescope, which can only observe partial transits. Future space-based missions designed to look at extrasolar planets, such as COROT and Kepler, will also have the ability to detect additional low-mass planets in transiting systems via transit timing analysis. For the time being MOST serves as a demonstration of the types of results that can be obtained from these types of observations.

Our results show no transit timing variations on three scales: (1) no long-term change in $P$ since 2003 at the $25 \mathrm{~ms}$ level, (2) no trend in transit timings during the 2005 run, and (3) no individual transit timing deviations above the $80 \mathrm{~s}$ level. The lack of variations on scales 2 and 3 helps exclude the presence of sub-Earth mass planets in inner resonant orbits and Earths and super-Earths in outer resonances and close-in $(P<10$ days $)$ orbits. Therefore, our results complement previous searches for a putative longer period planet, e.g., the 84 day period $0.127 M_{\text {Jup }}$ perturber proposed by Bodenheimer et al. (2003). Our TTVs have no sensitivity to detect a planet like that, but the radial velocity data have already ruled this out (Laughlin et al. 2005b). Through a combination of the 15 MOST transit times from 2004 and 2005 along with the 13 previously published transit times for HD 209458b obtained in 2000-2003 (Agol \& Steffen 2007) we have placed the most stringent limits on additional planets in this system with orbits between 1.5 and 10 days.

The level of orbital eccentricity of HD 209458b required to explain its anomalously large radius ranges between 0.012 and 0.03 , given the uncertainty range for Jupiter's tidal dissipation factor, $Q_{\mathrm{Jup}}$. In order to induce such orbital eccentricity, a companion planet would either need to be (1) significantly larger than Earth mass and in an outer orbit or (2) at least Earth mass and in a mean-motion resonance with the transiting planet. The first of these two cases is ruled out by radial velocity data (Laughlin et al. 2005b). The second case is ruled out by the TTV data. Hence, the problem of the radius of HD 209458b still remains, with the presence of a sufficiently perturbing companion planet in the system ruled out and obliquity tides remaining as an attractive alternative explanation.

Our conclusions supporting the lack of additional planets in this system are further corroborated by the work of Croll et al. (2007), who use MOST photometry of the HD 209458 system to rule out the presence of additional transiting planets with radii of 2-3 times that of the Earth and with periods ranging from 0.5 days to 2 weeks. While such planets would need to have suitable inclination angles to even be observed, the merit of a dual-transiting system is that the combination of the radii of the planets (given by the transit light curve) along with their masses (determined by their mutual interactions as seen in the TTVs) allows for the density of both planets in the system to be determined unequivocally (Holman \& Murray 2005). This application of the transit timing method could potentially allow for the first determination of the mass and density of an extrasolar Earth-like planet, since current RV precision cannot detect such planets.

We would like to thank Heather Knutson and Matt Holman for valuable input into determining and interpreting transit timing variations. We would also like to thank our referee, Eric Agol, for his very useful comments on this paper. J. M. M., D. B. G., A. F. J. M., S. M. R., and G. A. H. W. would like to acknowledge funding from the Natural Sciences and Engineering Research Council (NSERC), Canada. R. K. is supported by the Canadian Space Agency. W. W. W. would like to thank the Austrian FFG (project MOST) and FWF (P17580) for funding.

\section{REFERENCES}

Croll, B., et al. 2007, ApJ, 658, 1328

Guillot, T., \& Showman, A. P. 2002, A\&A, 385, 156

Henry, G. W., Marcy, G. W., Butler, R. P., \& Vogt, S. S. 2000, ApJ, 529, L41

Heyl, J. S., \& Gladman, B. J. 2007, MNRAS, 377, 1511

Holman, M. J., \& Murray, N. W. 2005, Science, 307, 1288

Knutson, H. A., Charbonneau, D., Noyes, R. W., Brown, T. M., \& Gilliland, R. L. 2007, ApJ, 655, 564 
Landsman, W. B. 1993, in ASP Conf. Ser. 52, Astronomical Data Analysis Software and Systems II, ed. R. J. Hanisch, R. J. V. Brissenden, \& J. Barnes (San Francisco: ASP), 246

Laughlin, G., Butler, R. P., Fischer, D. A., Marcy, G. W., Vogt, S. S., \& Wolf, A. S. 2005a, ApJ, 622, 1182

Laughlin, G., Marcy, G. W., Vogt, S. S., Fischer, D. A., \& Butler, R. P. 2005b, ApJ, 629, L121

Laughlin, G., Wolf, A., Vanmunster, T., Bodenheimer, P., Fischer, D., Marcy, G., Butler, P., \& Vogt, S. 2005c, ApJ, 621, 1072

Mandel, K., \& Agol, E. 2002, ApJ, 580, L171

Matthews, J. M., Kusching, R., Guenther, D. B., Walker, G. A. H., Moffat, A. F. J., Rucinski, S. M., Sasselov, D., \& Weiss, W. W. 2004, Nature, 430, 51
Miralda-Escudé, J. 2002, ApJ, 564, 1019

Radhakrishnan, K., \& Hindmarsh, A. C. 1993, LLNL Rep. UCRL-ID-113855

Rowe, J. F., et al. 2006, ApJ, 646, 1241

2007, ApJ, submitted (arXiv: 0711.4111)

Sartoretti, P., \& Schneider, J. 1999, A\&AS, 134, 553

Sasselov, D. D. 2003, ApJ, 596, 1327

Steffen, J. 2006, Ph.D. thesis, Univ. Washington

Steffen, J. H., \& Agol, E. 2005, MNRAS, 364, L96

Walker, G., et al. 2003, PASP, 115, 1023

Winn, J. N., \& Holman, M. J. 2005, ApJ, 628, L159

Zhou, J.-L., Aarseth, S. J., Lin, D. N. C., \& Nagasawa, M. 2005, ApJ, 631, L85 